Wentworth, H. W.-A quantitative study of achromatic and chromatic sensitivity from centre to periphery of the retina. Psychol. Monog., Vol. XL, 189 pp., 1930.

11. Ferree, C. E., Rand, G., and Monroe, M. M.-Studies in perimetry. I. Preliminary work on a diagnostic scale for the form field. Amer. Jl. of Ophthal., Vol. IX, pp. 95-104, 1926.

Studies in perimetry. II. Preliminary work on a diagnostic scale for the colour fields. Ibid., Vol. XII. pp. 269-285, 1929.

Studies in perimetry. IV. Preliminary work on a diagnostic scale for the form field with a 0.17 degree stimulus. Ibid., Vol. XIII, pp. 859-867, 1930.

Diagnostic scales for the 1 degree and 0.17 degree form field stimuli for the eight principal meridional quadrants taken separately. Arch. of Ophthal., Vol. VI, pp. 518-534, 1931.

Critical values for the limits of the colour fields in the eight principal meridional quadrants taken separately. Amer. Jl. of Ophthal., Vol. XVI, pp. 577-589, 1933.

\title{
THE RELATIONSHIP BETWEEN CONJUNCTIVITIS AND TRACHOMA*
}

\author{
BY \\ A. F. MacCallan, C.B.E. \\ PRESIDENT OF THE INTERNATIONAL ORGANIZATION \\ AGAINST TRACHOMA
}

I HAVE been requested to address this General Assembly of the International Association for the Prevention of Blindness and of the International Organization against Trachoma on "The Relationship between Conjunctivitis and Trachoma."

The term conjunctivitis is applied to any form of inflammatory change affecting the conjunctiva, while trachoma is a variety of conjunctivitis, the full name of which is conjunctivitis trachomatosa.

In order to recognise trachoma a definition is required, and I give the following formula :

Trachoma is a specific contagious disease of the conjunctiva in man. It is chronic in nature. It is characterized by a subepithelial infiltration of the conjunctiva by a cellular exudate, which spreads to the cornea and to the tarsus. It. is followed by cicatricial changes in the affected tissues.

That the disease is specific and contagious has been proved over and over again by the experimental or accidental inoculation of a healthy human conjunctiva from that of a trachomatous individual. The disease is chronic, such acute manifestations as occur being due, in the majority of cases, to superimposed

\footnotetext{
* Presidential Address at the session of the League against Trachoma, held in Paris, May 9-15, 1936.
} 
bacterial infections. However, occasionally trachoma gives the impression of beginning acutely; but this is due to the late diagnosis of the disease, which has been present for some time without being recognized; when discomfort is produced by friction of the roughness of the trachomatous conjunctiva on the sensitive cornea, the ophthalmologist is led to evert the upper eyelid and discover the condition. In heavily trachomatized countries the disease is frequently discovered during the course of, or during convalescence from, an attack of acute conjunctivitis. But as the late Charles Nicolle has said, "The acute conjunctivitis is the occasion of the diagnosis and not the occasion of the disease." The majority of the inhabitants of these countries become infected during the first two years of life. Born of trachomatous mothers it can only be by a miracle that infection will be avoided.

A subepithelial infiltration of the conjunctiva is present in every case of active trachoma; the round cells of which the infiltration is composed may be aggregated into clumps or "follicles," but there may be no follicular formation. I have seen thousands of young children in Southern Egypt, where the first stage of trachoma showed a reddened and thickened conjunctiva to the naked eye, but no follicles at all. The infiltration rapidly spreads in the superficial layer of the palpebral conjunctiva to the upper fornix; and from there it travels in the bulbar conjunctiva to the corneal limbus, and it is at this position, at the upper fifth of the corneal circumference, in tissue which is normally clear and avascular, that the first pathognomonic sign of trachoma appears.

The cellular invasion of the normally clear cornea appears first as an indefinite grey infiltration at the limbus. From the convexity of the end capillary loops of the conjunctiva new vessels are developed which run for a short distance towards the centre of the cornea. The cellular infiltration and the new vessels are inserted between the corneal epithelium and Bowman's membrane, and together form the pathognomonic sign of early trachoma, known as pannus.

This phenomenon of infiltration and vascularization of the normally clear corneal periphery in the earliest stage of trachoma requires intense illumination as well as optical magnification for recognition. This is best secured by the use of the slit-lamp. Every doubtful case of trachoma requires examination by this means.

In severe or long-standing cases of trachoma the cellular infiltration between the corneal epithelium and Bowman's membrane partially destroys the latter, when the infiltration penetrates between the layers of the corneal stroma. Marked opacity is thus produced, which becomes permanent on conversion of the inflam- 
matory exudate into cicatricial tissue. The folly of an attempt "to cure pannus" therefore is apparent.

The trachomatous changes which occur in the tarsus are first a blocking of the ducts of the Meibomian glands, leading to the bleb-like excrescences of the second stage of the disease, (Tr. II a), which emit a gelatinous material when roughly handled; and then to a wide-spread infiltration of the tarsus with inflammatory cells, which becoming resolved into connective tissue, leads to thickening of the structure and entropion.

So that among inflammatory affections of the conjunctiva trachoma is absolutely unique both in its clinical manifestation, pannus, and in the pathological changes which occur as the result of the disease.

In order to appreciate the difference between trachoma and other forms of conjunctivitis the evolution of the former requires consideration. There are four stages, which are divided according to the comparative prominence of subepithelial lymphoid infiltration, bleb-like excrescences, and connective tissue formation.

In the first stage, $\mathrm{Tr}$. I, after an incubation period of from 4 to 10 days a subepithelial infiltration appears, which is usually, but not always, concentrated into tiny greyish islets, the so-called follicles, which, however, are not pathognomic. For diagnosis reliance must be placed on slit-lamp detection of corneal vascularization characteristic of trachoma. In babies and young children it may be quite impossible to use the slit-lamp; in a trachomatous country an infant with a red and velvety conjunctiva may receive the diagnosis of "trachoma dubium," as also must many other cases if no slit-lamp is available.

This stage may be converted into the second stage, or may develop into the third stage directly. On rare occasions both conjunctiva and cornea may return to normal, bearing no trace of the former condition.

The second stage, Tr.II, has been divided into sub-groups for application to different appearances which are common in the East. For present purposes, only one need be mentioned, Tr. II $a$. Here there are the bleb-like excrescences, which are pathognomonic of trachoma. Pannus is invariably present.

In the third stage, 'Tr. III, the inflammatory exudate is beginning to be replaced by scar-tissue, and there is thickening of the tarsus. Islands of inflamed conjunctiva are seen to be surrounded by a cicatricial network. Here again the condition is typical, and is not imitated by any other form of conjunctivitis. There is always pannus.

In the fourth stage, $\mathrm{Tr}$. IV, all inflammatory changes have come to an end. The conjunctiva is covered by scar-tissue 
epithelium and there is more or less cicatricial change in the thin sub-epithelial tissue and in the tarsus. Entropion due to tarsal changes and trichiasis due to proliferation of hair follicles may be present, both of which conditions are pathognomonic.

It is therefore clear that in the first stage of trachoma only, is there any difficulty in making a diagnosis between this disease and other forms of conjunctivitis. This difficulty is resolved by the detection of cellular infiltration and vascularization of the normally clear cornea at the upper fifth of its circumference.

There are no chemical or microscopical tests which, when applied to the conjunctival secretion, assist in making a diagnosis; nor would assistance be obtained by removing a portion of the conjunctiva for microscopical examination. In some cases, but not in all, a light scraping of the conjunctiva removes some of the superficial cells, which, when stained with Giemsa, exhibit the cellular inclusions described by Halberstaedter and Prowaczek; but these are found in conditions other than trachoma, such as in swimming-bath conjunctivitis, and in the non-gonococcal conjunctivitis of infants; nor can they invariably be detected in the first stage of trachoma; it is quite useless to search for them in the second stage.

This essay on "The Relationship between Conjunctivitis and Trachoma" cannot be concluded without an enumeration of the various forms of conjunctivitis which, on occasion, may bear some resemblance to trachoma. They may be divided into common conditions, uncommon conditions, rare conditions, and tropical conditions. They are as follows :-

\section{Common Conditions.}

Follicular conjunctivitis in children.

Acute conjunctivitis with follicles.

Chronic conjunctivitis with corneal vascularization of former interstitial keratitis, or former phlyctenular ulceration.

\section{UnCOMmon Conditions.}

Spring catarrh.

Toxic follicular conjunctivitis.

Parinaud's conjunctivitis.

Swimming-bath conjunctivitis with inclusions.

Conjunctivitis with inclusions not of swimming-bath origin.

Non-gonococcal conjunctivitis with inclusions in infants.

Follicular type of tuberculous conjunctivitis, or syphilitic conjunctivitis. 
Rare Conditions.

Actinomycosis.

Sporotrichosis.

Squirrel-plague conjunctivitis.

Bilateral lymphoid infiltration of the conjunctiva.

\section{Tropical Conditions.}

Trachoma dubium.

Chronic gonococcal conjunctivitis.

It is not necessary to describe the clinical manifestations which characterize each of the conditions of conjunctivitis enumerated above. It is only necessary to say that in the absence of infiltration of the normally clear cornea by a cellular exudate, and of the vascularization characteristic of trachoma, the condition under consideration may be determined to be non-trachomatous.

In conclusion I do not wish to give the impression that I minimize the value of ordinary clinical observation by an experienced trachomatologist in the diagnosis of conjunctival conditions. In many cases of follicular trachoma, stage I, the appearance of the follicles and their position at each extremity of the retrotarsal fold of the upper lid is characteristic. However, no such characteristic appearance obtains in trachoma when there are no obvious follicles, but merely a wide-spread subepithelial infiltration which makes the conjunctiva red and velvety.

\section{INCIDENCE OF MYOPIA IN CHINA}

Data and theses from periodical investigations eovectors thirty years residence, and association with refracting and hoopteal centres, in a score of the larger cities

BY

\section{O. D. RASMUSSEN}

General background.-A sharp distinction had to be made between ancient native refracting methods and treatment and the modern school, represented by comparatively recent medical missionary, private Western, and Western-educated Chinese enterprise.

Modern practices began in the last decades of the 19th Century, spreading rapidly from 1900 onwards. Ancient schools rejected modern ideas within their own craft and in the past tan years 